\title{
LncRNA GAS5 Represses Osteosarcoma Cells Growth and Metastasis via Spor ing MiR-203a
}

\author{
Yang Wang Daliang Kong
}

Department of Orthopedics, China-Japan Union Hospital of Jilin University,

Key Words

Osteosarcoma - LncRNA GAS5 - MiR-203a signaling

\section{Abstract}

Background/Aims: LncRNA GAS5, a tumor actions in various cancers, wh action is still unclear. This stydy wa osteosarcoma and tried to IncRNA GAS5 in MG-63 cells by vector transfection. assessed by MTT, Transw miR-203a, as well as bet dual luciferase activity as.

Osteosarcoma, also known as osteogenic sarcoma, is a primary osteogenic tumor with a high degree of malignancy, characterizing by tumor cells directly form bone-like tissue [1]. Osteosarcoma is most prevalent in young people under the age of 20. Surgery is one of the main treatments for patients with osteosarcoma. Despite the success of surgery for 


\section{Cellular Physiology and Biochemistry Published \begin{tabular}{l|l} 
DOI: 10.1159/000487178 & $\begin{array}{l}\text { () } 2018 \text { The Author(s). Published by S. Karger AG, Basel } \\
\text { www.karger.com/cpb }\end{array}$
\end{tabular} \\ Wang et al.: Effect of LncRNA GAS5 on Osteosarcoma Cells}

osteosarcoma, the 5-year overall survival rate of patients with metastatic or recurrent osteosarcoma remains dismally poor [2]. This calls for a better understanding of the pathogenesis and molecular mechanisms of osteosarcoma, which will helpful in the clinical treatment and prognosis of this disease.

Long non-coding RNAs (lncRNAs) are a category of non-protein encoding RNAs wi longer than 200 nucleotides. LncRNAs are previously considered as "dark matter" of th transcription [3], but recently most of the known lncRNAs have been reported to be critical in a number of biological and pathological processes, like cell proliferation, migration, invas apoptosis and differentiation. The functions of IncRNAs in regulating gene gxpressic human cancers have been gradually uncovered. LncRNA growth arrest-specif (GAS5), a suspensor in cell growth, has been reported to participated in the deve multiple cancers, including colorectal cancer [4], non-small-cell lung can cancer [6]. Specifically, in cervical cancer, decreased expression of IncRNA with a poor prognosis [7]. An in vitro investigation revealed that silep induced a significant increase in pancreatic cancer cells proliferation studies demonstrated IncRNA GAS5 as a tumor suppressive gene: hon tho thious GAS5 in osteosarcoma is remain unclear.

It has been acceptable that IncRNAs implicate interacting miRNAs [9]. miRNAs are another kind of non ing with organism development and various human diseases. Th and miRNA is not entirely clear yet [10], but se studies hav and miRNA is not entirely clear yet [10], but se studies hat dicated that lncRNAs have by binding effect, thereby exerting tumor-pro or tumor-suppressive activities in multiple kind of cancers [12]. The lncRNA-mi A-m interactions may be conducive to understand the mechanism and pathogenesis rnce

In this study, we aimed to inve ctional effect of IncRNA GAS5 on osteosarcoma cells growth and met as Additronally, we focused on the interaction between IncRNA GAS5 and miR-203a between miR-203a and a miR-203a target gene TIMP2. The findings is helpful for enlarging our insight into the
pathogenesis of osteosarcom
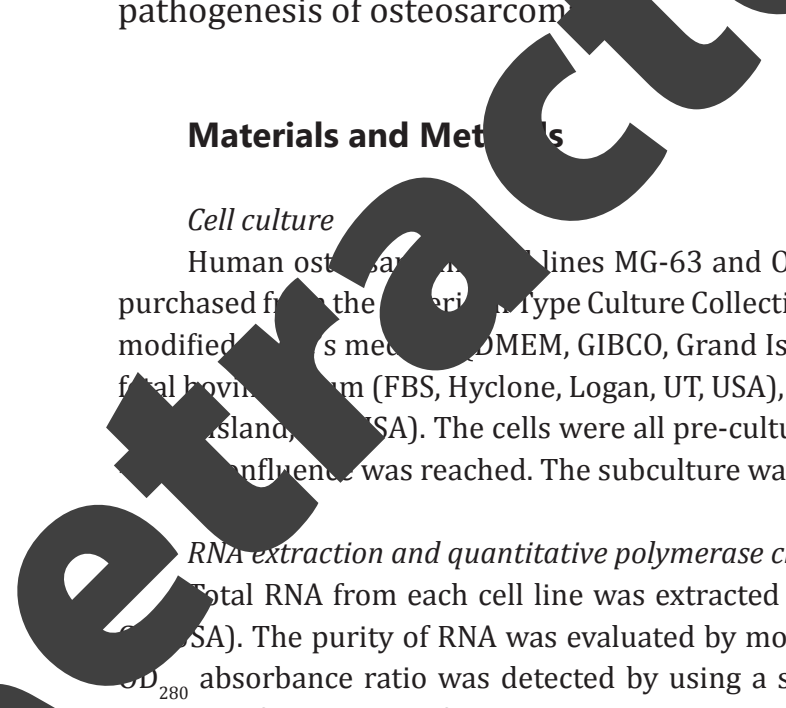

\section{f al bovil (FBS, Hyclone, Logan, UT, USA), $100 \mathrm{U} / \mathrm{ml}$ penicillin and $100 \mathrm{U} / \mathrm{ml}$ streptomycin (GIBCO, sland, SA). The cells were all pre-cultured in a humidified incubator at $37^{\circ} \mathrm{C}$ with $5 \% \mathrm{CO}_{2}$ until nflmen was reached. The subculture was collected for use in this study.}

Yines MG-63 and OS-732, and a human osteoblast cell line hFOB1.19 were
Human ost Culture Collection (ATCC, Rockville, MD, USA) and cultured in Dulbecco's

$R N A$ extraction and quantitative polymerase chain reaction ( $q P C R$ )

otal RNA from each cell line was extracted by using Trizol (Invitrogen Life Technologies, Carlsbad, SA). The purity of RNA was evaluated by modified formaldehyde agarose gel electrophoresis. $\mathrm{OD}_{260} /$ absorbance ratio was detected by using a spectrophotometer (Genova Nano, BIBBY, UK). The One Step SYBR ${ }^{\circledast}$ PrimeScript ${ }^{\circledast}$ PLUS RT-RNA PCR Kit (TaKaRa Biotechnology, Dalian, China) was used for qPCR analysis to test the expression levels of GAS5 and TIMP2. The Taqman MicroRNA Reverse Transcription Kit and Taqman Universal Master Mix II (Applied Biosystems, Foster City, CA, USA) were used for testing the expression of miR-203a. The primers were synthesized by GenePharma (Shanghai, China). The expressions of GAS5 and TIMP2 were normalized to GAPDH, and miR-203a expression was normalized to U6 snRNA. Fold changes were calculated by $2^{-\Delta \Delta C t}$ method. The data were analyzed by Real-Time StatMiner (Integromics, Granada, Spain) [13].

\section{KARGER}




\section{Cellular Physiology Cell Physiol Biochem 2018;45:844-855 \begin{tabular}{l|l|l|l} 
DOI: 10.1159/000487178 & O 2018 The Author(s). Published by S. Karger AG, Basel \\
www.karger.com/cpb
\end{tabular} \\ Wang et al.: Effect of LncRNA GAS5 on Osteosarcoma Cells}

\section{Cell transfection}

The specific short-hairpin RNA (shRNA) against human lncRNA GAS5 was cloned into pENTR ${ }^{\mathrm{TM}}$ / U6 plasmid (GenePharma, Shanghai, China), and referred as sh-GAS5. A non-targeting shRNA (shNC, GenePharma) was used as a negative control. The full-length of IncRNA GAS5 sequence was transfected into pcDNA $^{\mathrm{TM}} 3.1$ vector (ThermoFisher, Scientific, China) and referred as pc-GAS5. The empty pcDNA? vector was used as a negative control. miR-203a mimic, inhibitor or their respective scramble controls we. synthesized by RiboBio (Guangzhou, China). TIMP2 specific siRNA (si-TIMP2) and siRNA negative control were purchased from Santa Cruz (Santa Cruz Biotechnology, USA). For TIMP2 overexpressing, the full-le of TIMP2 sequence was transfected into $\mathrm{pcDNA}^{\mathrm{TM}} 3.1$ vector (ThermoFisher, Scientific, Chin 2 and ref as pc-TIMP2. All transfections were performed by using Lipofectamine 3000 reagent (L Corporation, Carlsbad, CA, USA) according to the manufacturer's protocol. The stably trans cells were selected by using the culture medium containing $0.5 \mathrm{mg} / \mathrm{ml} \mathrm{G418}$ (SigmaUSA). The stable transfected cells were selected for the subsequent experiments.

Cell viability assay
After transfection, cell viability of stable transfected MG-63 cells 5-dimethylthiazol-2-yl)-2, 5-diphenyltetrazolium bromide (MTT) col atric assà sfected cells were seeded in 96-well plates at the density of $2 \times 10^{3}$ cells/well. After 1 Aldrich, St. Louis, MO, USA) was added into each well and incubate an $\quad$ at $37^{\circ} \mathrm{C}$. Then, $150 \mu \mathrm{l}$ dimethylsulfoxide (DMSO, Sigma-Aldrich, St. Louis, MO, USA) was add neres were shaken for 10 min. The absorbance was measured at $570 \mathrm{~nm}\left(\mathrm{OD}_{570}\right) \mathrm{g} 680$ micro enzym-linked immunosorbent assay (ELISA) reader (Bio-Rad Laboratories, Hemel Hed UK). Cell vas ty was expres a ratio of the control group and calculated as the absorbance

\section{Migration and invasion assay}

Cell migration was determined by using hamber migration assay chamber with a pore size of $8 \mu \mathrm{m}$ polycarbonate membran cornıng, New York, USA). In brief, MG-63 cells after transfection were suspended in $200 \mu \mathrm{l}$ ser of 24-well chamber. $600 \mu \mathrm{l}$ of ce added into the lower compartment. After $12 \mathrm{~h}$ of incubation at $37^{\circ} \mathrm{C}$, cells were fixed vethan or (1ST, USA) for $30 \mathrm{~min}$. Non-traversed cells were removed from the upper surface of the rang a cotton swab. Traversed cells on the lower chamber were stained with $0.1 \%$ cry violet (__o, USA). To quantify migration, the cells in per well were counted from five independ magnification.

The invasion Muros cells after transfection was determined by using a BD BioCoat ${ }^{\mathrm{TM}}$ Matrigel $^{\mathrm{TM}}$ Invas $8 \mu \mathrm{m}$ polycarbonate membrane) supplemented with matrigel. Briefly, cells were treate $\mathrm{r}$ ind io mdition, and $5 \times 10^{4}$ cells in $200 \mu \mathrm{l}$ serum-free medium were seeded on the upper co ment wellates, while complete medium containing 10\% FBS was added into the lyeracha. The invasion chambers were incubated for $48 \mathrm{~h}$ in the incubator with $5 \% \mathrm{CO}_{2}$ at $37^{\circ} \mathrm{C}$. The (NIS , SA) and stained by crystal violet solution (Amresco, USA). To quantify invasion, the cells

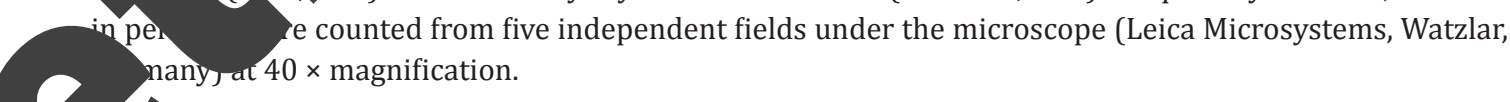

After transfection, early apoptosis was detected by using Annexin V- PE Apoptosis Detection Kit (South Biotech, USA). MG-63 cells in culture medium were washed with phosphate buffered saline (PBS) and fixed with 70\% ethanol (Sigma-Aldrich, St. Louis, MO, USA), then washed twice again and stained in propidium iodide (PI)/ fluorescein isothiocynate (FITC) conjugated Annexin V with the presence of $50 \mu \mathrm{g} /$ $\mathrm{ml}$ RNase A (Sigma-Aldrich, St. Louis, MO, USA). The samples were incubated in the dark for $1 \mathrm{~h}$ at room temperature. The early apoptotic cells (Annexin-V positive and PI negative) were detected by using FACSCan (BD Biosciences, USA). All the measurement was performed in triplicate and data was analyzed by FlowJo Version 10.0 software (FlowJo LLC, USA).

\section{KARGER}




\section{Cellular Physiology

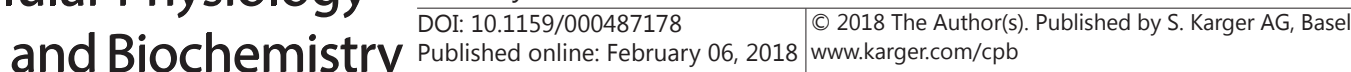 \\ Wang et al.: Effect of LncRNA GAS5 on Osteosarcoma Cells}

Dual luciferase activity assay

The sequence of TIMP2 3'-UTR containing the predicted miR-203a binding site was cloned into pGL2Basic Vector (E1641, Promega, WI, USA) as the reporter vector TIMP2-wild-type (TIMP2-wt). Mutated TIMP2 3'-UTR was used as a negative control, namely TIMP2-mutated-type (TIMP2-mt). Each vector was co-transfected with miR-203a mimic into HEK 293T cells. The cells were collected $48 \mathrm{~h}$ later, and the Fire and Renilla luciferase activity were measured by using the Dual-luciferase reporter system (Promeg Madison, WI, USA) according to the manufacturer's instructions.

\section{Western blot assay}

The protein samples were extracted by using RIPA Lysis Buffer (Beyotime Biotechn China) supplemented with protease inhibitors (Roche, Basel, Switzerland) and quantified Protein Assay Kit (Pierce, Appleton, WI, USA). Western blot system was established usi Gel system according to the manufacturer's instructions. All blots were transferred on, fluoride (PVDF) membrane and incubated with primary antibodies overnight at $4^{\circ} \mathrm{C}$. with the dilution of 1:1000 in this step were as follows: TIMP2 (sc21735), B $\mathrm{c}$ sc509), Bax (sc20067), Caspase-3 (sc7272), Caspase-9 (sc17784), nuclear factor gene enhancer in B-cells inhibitor, alpha ( $\mathrm{I \kappa B} \alpha, \mathrm{sc847})$, phosphor $\mathrm{I \kappa B} \alpha(\mathrm{sc101713}), \mathrm{p} 65$ (sc109) and phosphorylated p65 (p-p65, sc101749) (Santa Cruz, D1 (a) 875), Cyclin B1 (ab32053), Cyclin-dependent kinase 1 (CKD1, ab133327), CKD4 (ab 357 Caspase-3 (ab2302), cleaved-Caspase-9 (ab2324), PI3K (ab86714), phosphorylated PI3K , ầ _2651), AKT (ab8805), phosphorylated AKT (p-AKT, ab38449), Glycogen syntb_kinase 3 beta _ 3 $\quad$, ab32391), phosphorylated GSK3 $\beta$ (p-GSK3 $\beta$, ab75745) and GAPDH (ab8245) (Ab VSA). Then nembranes were washed and incubated with secondary antibodies marked by horser. roxidase (ARP, 1:5000; Santa Cruz) for $1 \mathrm{~h}$ at room temperature. The membranes were transfec nto hemiDoc ${ }^{\mathrm{TM}} \mathrm{XRS}$ system (Bio-Rad, USA), and $200 \mu \mathrm{l}$ Immobilon Western Chemiluminescent $\mathrm{H}$ ubst Merck Millipore, MA, USA) was added. The signals were captured and the intensity fuantified by using Image Lab ${ }^{\text {TM }}$ Software
(Bio-Rad, Hercules, CA, USA).

\section{Statistical analysis}

All experiments were repea timesene results of multiple experiments were presented as mean \pm standard deviation ( $S^{P}$ Saa a (GraphPad Software, San Die A, USA). -values were calculated using a one-way analysis of variance (ANOVA) for multiple grol and ty considered to indicate ctatis

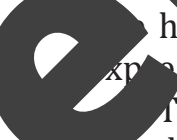

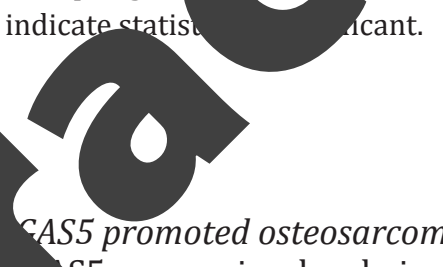

AS5 promoted osteosarcoma cells growth and metastasis

CR 55 expression levels in different cell lines were determined by qPCR. The in Fig. A showed that lncRNA GAS5 expression levels were significantly suppressed teosarcoma cell lines MG-63 and OS-732 compared with human osteoblast cell hFow1.19 $(P<0.01)$. Since MG-63 cells have the sharpest decrease in lncRNA GAS5
ssion, MG-63 cell line was selected for use in the subsequent experiments.

then, the transfection efficiency of MG-63 cells transfected with IncRNA GAS5 shRNA as determined by qPCR. The results in Fig. 1B showed that the expression of lncRNA GAS5 was clearly inhibited compared with shNC control $(P<0.001)$. MTT assay results (Fig. 1C) suggested that cell viability was improved by transfection with sh-GAS5 compared with transfection with shNC $(P<0.05$ or $P<0.001)$. Moreover, the expressions of Cyclin D1, Cyclin B1, CDK1 and CDK4 were all up-regulated in sh-GAS5 group than in shNC group (Fig. 1D and 1E). Transwell detection results in Fig. 2A-2D showed that, MG-63 cells migration and invasion were both enhanced after sh-GAS5 transfection $(P<0.05)$.

The impacts of IncRNA GAS5 overexpression on MG-63 cells growth and metastasis were also detected. In Fig. 3A, lncRNA GAS5 expression was significantly increased by transfection

\section{KARGER}


Fig. 1. Effect of IncRNA GAS5 silence on MG-63 cells proliferation. (A) The expression of IncRNA GAS5 in human osteosarcoma cell lines (MG-63 and OS-732) and a human osteoblast cell line hFOB1.19 were detected by qPCR. (B) The RNA level expression of IncRNA GAS5 in MG-63 cells after transfection with sh-GAS5 and shNC were measured by qPCR. (C) Cell viability after transfection was detected by MTT assay. (D) Quantitative analysis of proliferation-related protein expressions based on western blot analysis. (E) Representative image of western blot analysis. *, $\mathrm{P}<0.05$, **, $\mathrm{P}<0.01$ and $* * *, \mathrm{P}<0.001$ compared to hFOB1.19 cells or shNC group.

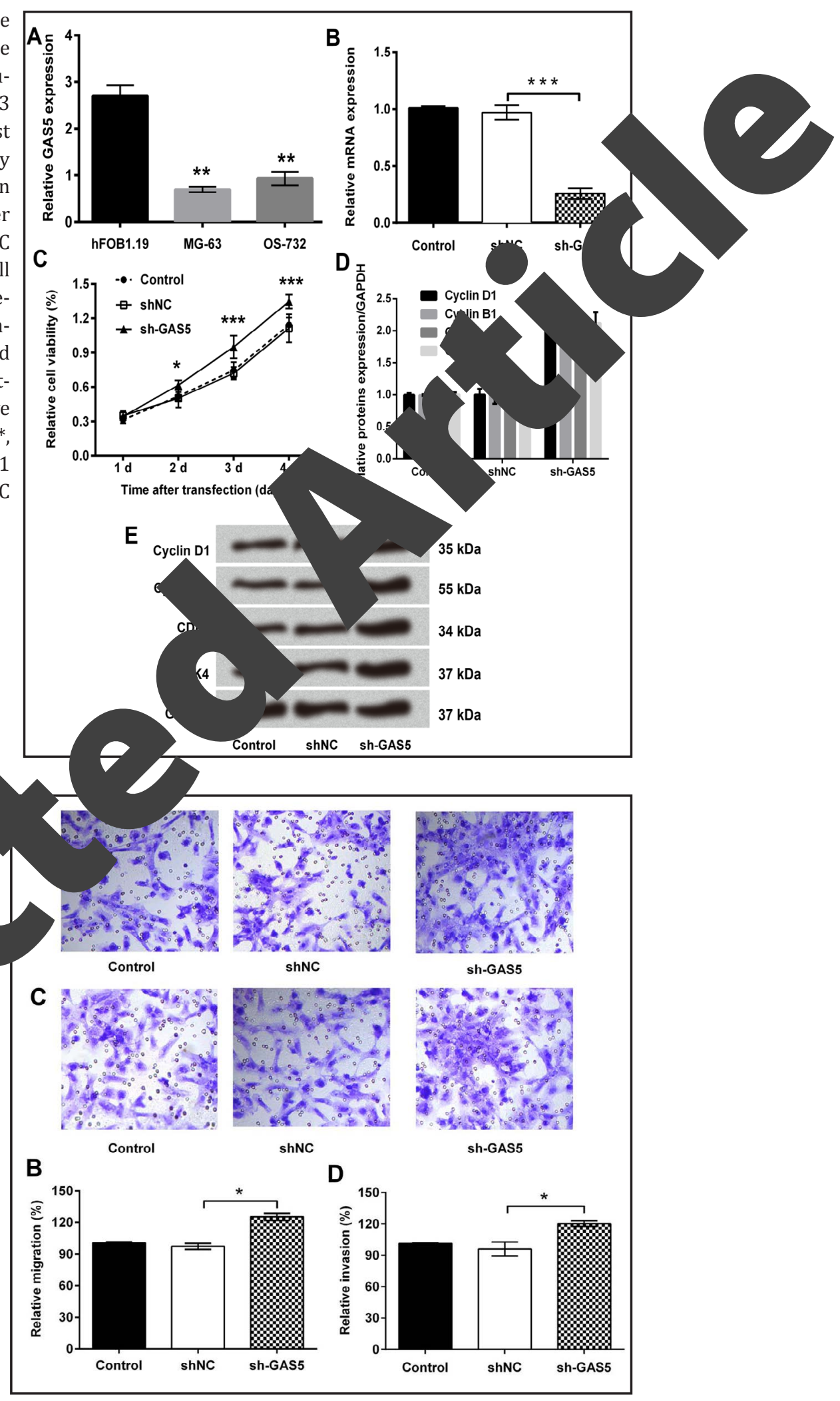

Fig. 2. Effects of lncRNA GAS5 lence on MG-63 cells migration an invasion. (A-B) Transwell was performed to measure tion after MG-63 cells were fected with sh-GAS

D) Transwell ass

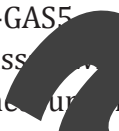
$\mathrm{P}<0.05$ com $\mathrm{gd}$ group.
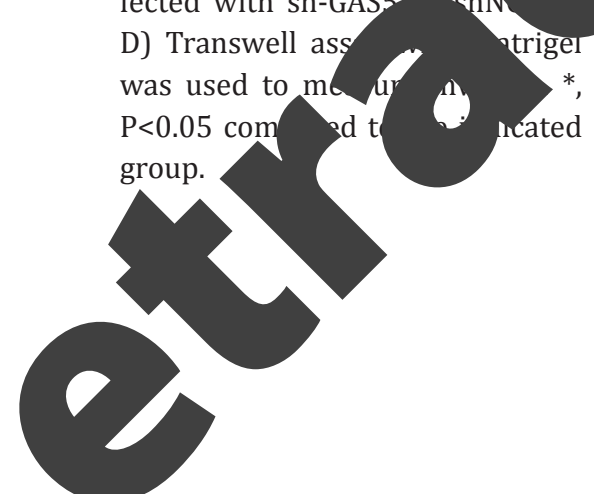

with pc-GAS5 (a GAS5 expressing vector) when compared with its negative control pcDNA3.1 $(P<0.001)$. Cell viability was significantly decreased $(P<0.05$ or $P<0.001$, Fig. 3B), and Cyclin D1, Cyclin B1, CDK1 and CDK4 expressions were remarkably down-regulated by pcKARGER 
Fig. 3. Effects of lncRNA GAS5 overexpression on MG-63 cells proliferation, migration and invasion. (A) The RNA level of IncRNA GAS5 was tested by qPCR in MG63 cells after transfection with pc-GAS5 or pcDNA3.1. (B) Cell viability was detected by MTT assay. (C) Expression changes of proliferation-related protein were measured by western blot. (D) Transwell assay was performed to measure cell migration. (E) Transwell assay with matrigel was used to measure cell invasion. ${ }^{*}, \mathrm{P}<0.05$ and ${ }^{* * *}, \mathrm{P}<0.001$ compared to $\mathrm{pcD}$ NA3.1 group.

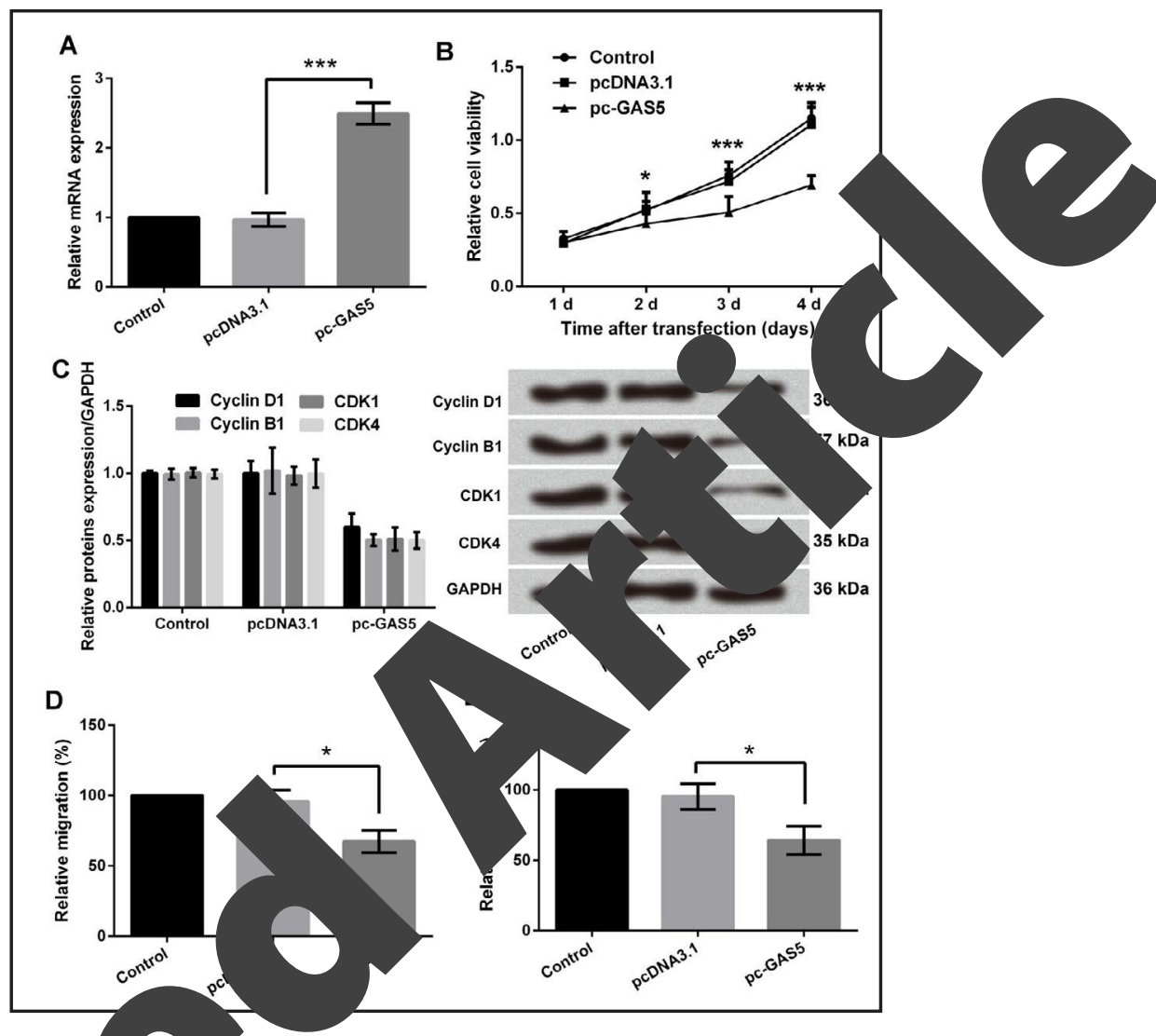

AS5 transfection (Fig. 3C). Meanwhile
were both decreased in the

LncRNA GAS5 affecte Mra expression

We firstly detected shown in Fig. 4A, ch-GA. exprion significantly increased the expren of miR-203a compared with gatrve control group $(P<0.001)$. Then, the expression of miR-203a in MG-63 cells ar sed by inhibitor transfection to see the involvement of miR-203a in MG-63 "s v that mi 3 a ex on was clearly down-regulated in miR-203a inhibitor transfected Din (1). Data in Fig. 4C-4G showed that, miR-203a inhibitor resulted in significant dses yiability, migration, invasion $(P<0.05$ or $P<0.01)$ and a significant increase totic $a$ rate $(P<0.001)$. More interestingly, sh-GAS5 induced the increases of cell ablion, invasion, the up-regulations of Cyclin D1, Cyclin B1, CDK1, and CDK4, the mnibition of cell apoptosis were all abolished or attenuated by miR-203a inhibitor.

Overexpression of TIMP2 decreased MG-63 cells growth and metastasis

Next, TIMP2 was overexpressed in MG-63 cells by vector expression to explore the role of TIMP2 in osteosarcoma. In Fig. 5A and 5B, up-regulations of TIMP2 at mRNA and protein levels were observed in MG-63 cells after transfection with pc-TIMP2 $(P<0.01)$. Cell viability, migration and invasion were reduced, while cell apoptosis was significantly increased in pcTIMP2 group compared with negative control group (Fig. 5C-5F, $P<0.05$ or $P<0.01$ or $P$ $<0.001$ ). Then, the apoptosis-related protein expressions were measured by western blot assay. As shown in Fig. 5G, the expression of Bcl-2 was obviously decreased, while Bax, cleaved caspase- 3 and cleaved caspase- 9 were increased when TIMP2 overexpression. 
Fig. 4. Involvement of miR-203a in IncRNA GAS5-modulated cell growth and metastasis of MG-63 cells. (A) The RNA levels of miR203a in cells transfected with shGAS5 or shNC. (B) The RNA levels of miR-203a in cells transfected with miR-203a inhibitor or inhibitor control. RNA levels of miR-230 were measured by qPCR. (C) Cell viability was detected by MTT assay in MG-63 cells after transfection with sh-GAS5, miR-203a inhibitor, sh-GAS5 plus miR-203a inhibitor or negative controls. *, $\mathrm{P}<0.05$ and ${ }^{* *}, \mathrm{P}<0.01$ comparison between sh-GAS5 and shNC + siNC group. \#, $\mathrm{P}<0.05$ and \#\#, $\mathrm{P}<0.01$ comparison between sh-GAS5 + miR-203a inhibitor and sh-GAS5 + siNC group. (D) Expression changes of proliferation-related proteins were measured by western blot. (E) Transwell assay was performed to measure cell migration. (F) Transwell assay with matrigel was used to measure invasion. (G) Apoptotic cells were measured by flow cytometry. *, $\mathrm{P}<0.05$, $\mathrm{P}<0.01$ and $* * *, \mathrm{P}<0.001$ compare to the indicated group.
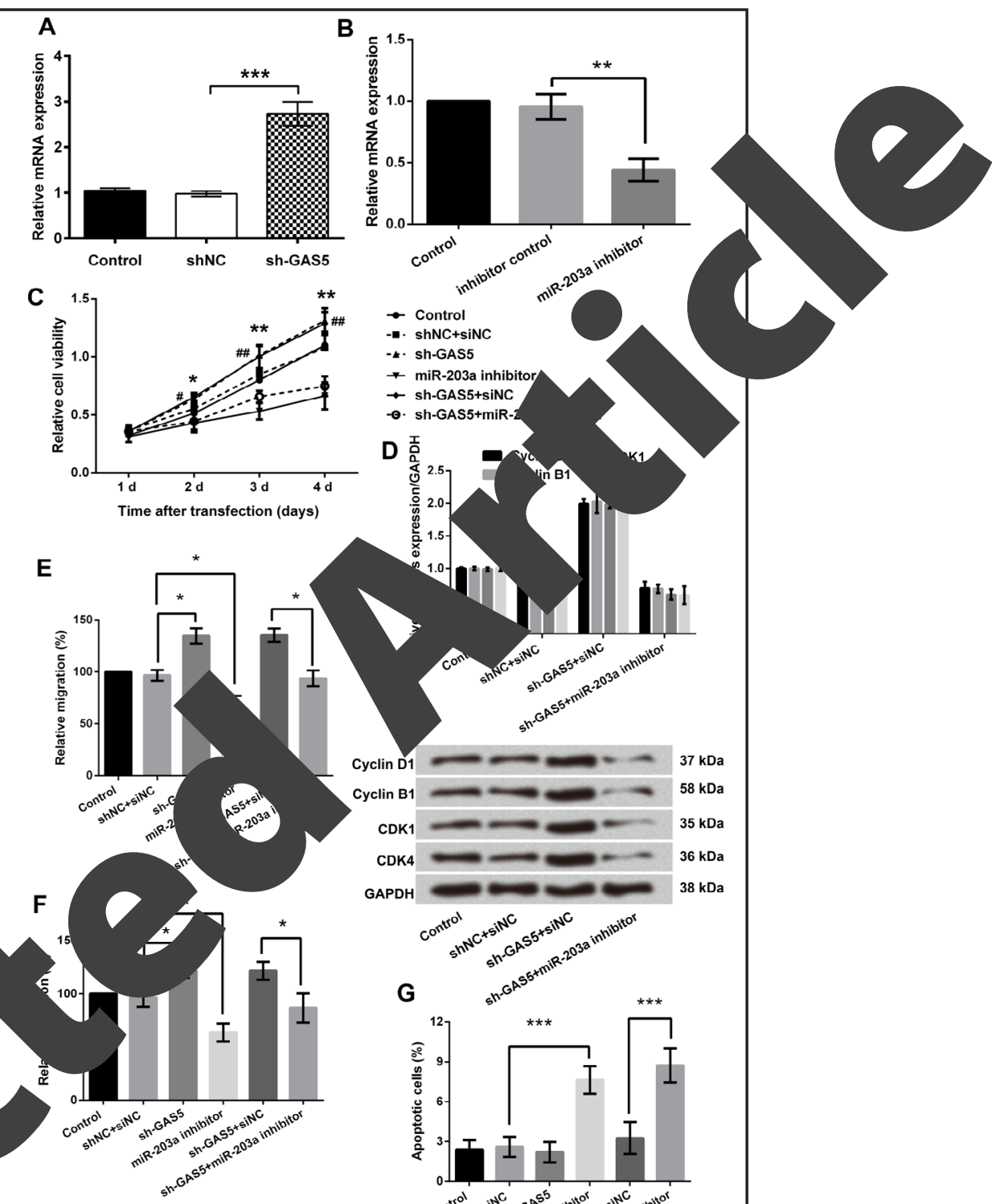

TIMP2 was a targe ne of miR-203a

MG-63

transfected mimic, inhi itor a so rble controls $x$ protein<smiles>[GeH2]=[TeH4]</smiles>
ression levels IP2 detected. As

in Fig. $A$ and 6B, miR-203a mimic transfection decreased the expression of TIMP2 in $b$ IA $(P<0.05)$ and protein levels, whereas miR-203a suppression significantly reasu IIMP2 expression $(P<0.01)$. Dual luciferase activity assay results in Fig. 6C su ating TIMP2 might be a target gene of miR-203a.

The involvement of TIMP2 in miR-203a-modulated growth and metastasis in MG-63 cells were detected then. The results of Fig. 7A suggested that miR-203a mimic transfection suppressed the expression of TIMP2 $(P<0.05)$, while miR-203a and TIMP2 simultaneous overexpression significantly reversed this trend $(P<0.01)$. MTT assay results in Fig. 7B showed that miR-203a mimic improved cell viability $(P<0.05)$, but co-transfection with miR-203a mimic and pc-TIMP2 significantly inhibited cell viability $(P<0.05$ or $P<0.01)$. The similar effects were found in cell migration and invasion. The results suggested that cell migration and invasion were enhanced by miR-203a mimic, whereas were reduced by miR-203a and TIMP2 simultaneous overexpression $(P<0.05$, Fig. 7C and 7D). The apoptosis 
Fig. 5. Effects of TIMP2 overexpression on MG-63 cells growth and metastasis. (A) The mRNA and (B) protein levels of TIMP2 were detected by qPCR and western blot respectively in MG-63 cells after transfection with pc-TIMP2 and pcDNA3.1. (C) Cell viability was measured by MTT assay. (D) Transwell assay was performed to measure cell migration. (E) Transwell assay with matrigel was used to measure invasion. (F) Apoptotic cells were measured by flow cytometry. (G) Expression changes of apoptosis-related proteins were measured by western blot. *, $\mathrm{P}<0.05$, ** $\mathrm{P}<0.01$ and $* * *, \mathrm{P}<0.001$ compared to pcDNA3.1 group.
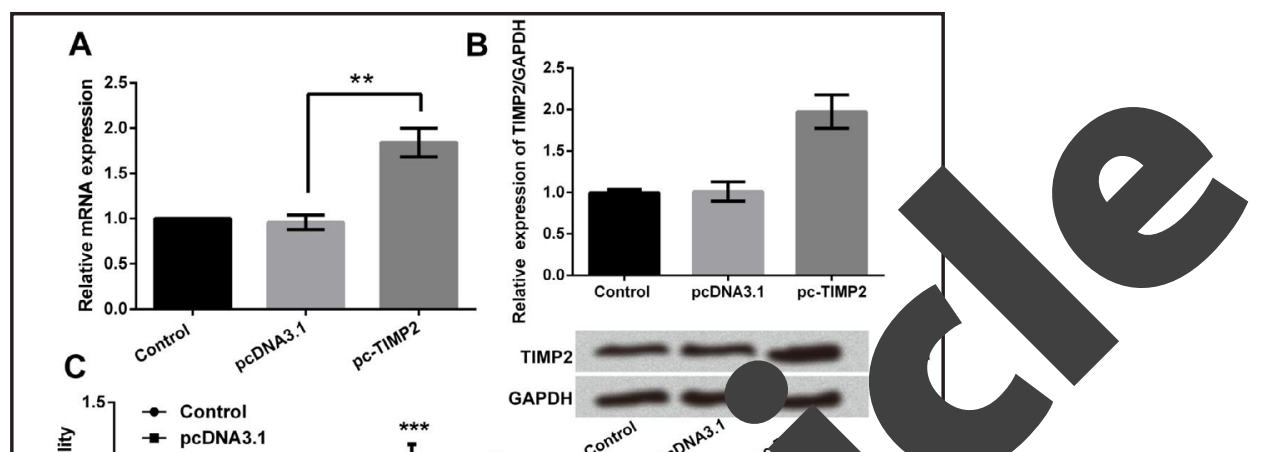

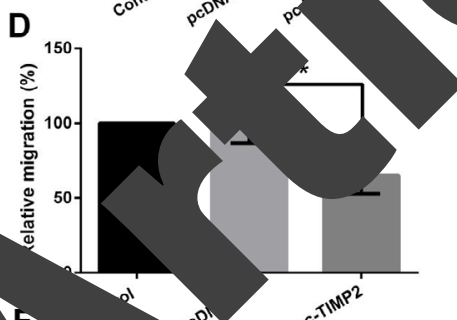

(

\section{ormed after transfection with}

IIMP2 3'UTR wild-type (wt) and miR-203a mimic, or TIMP2 3'UTR mutant-type (mt) and miR-203a mimic. ${ }^{*}, \mathrm{P}<0.05$ and $* *, \mathrm{P}<0.01$ compared to the indicated group.

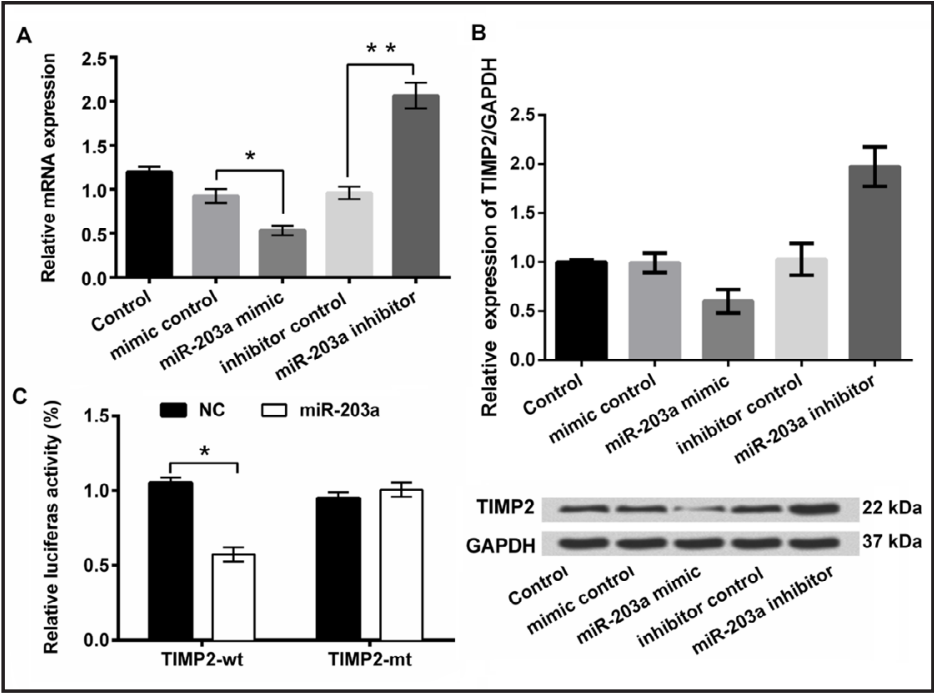


assay results (Fig. 7E) showed that miR-203a mimic plus pc-TIMP2 significantly increased the rate of apoptotic cells compared with miR-203a mimic alone $(P<0.01)$. From Fig. 7F, we found that the expression of Bcl2 was decreased, and Bax, cleaved caspase-3, cleaved caspase-9 were increased after miR-203a inhibitor transfection. However, co-transfection with miR-203a inhibitor and si-TIMP2 up-regulated Bcl-2, and down-regulated Bax, cleaved caspase-3, and cleaved caspase-9.

Further, the interaction between TIMP2 and IncRNA GAS5 were detected by qPCR and western blot. As shown in Fig. $7 \mathrm{G}$ and $7 \mathrm{H}$, the mRNA and protein expression levels of TIMP2 were up-regulated by pcGAS5transfection, on the contrary, sh-GAS5 down-regulated TIMP2 expression $(P<0.05$ or $P<0.01)$.

Regulatory role of IncRNA GAS5, miR-203a and TIMP2 in PI3K/ $A K T / G S K 3 \beta$ and NF- $\kappa B$ signaling pathways

The expressions of mai tors related with PI3K/AKT, and NF- $\mathrm{KB}$ signaling pathy detected by western blo rer cell were transfected witl iR-2 mimic, inhibitor, ch-GA

TIMP2, respec shown in Fig. 8A, the of a sed PI3K, AKT and K3B or mons were increas sh-c or miR-203a pimic, a creased by miR-203a or. 203a inhibitor indown regulations of phosPI3K, AKT and GSK3 $\beta$ e reversed by si-TIMP2 transfec-
From Fig. 8B, the phosphory$\mathrm{I} \kappa \mathrm{B} \alpha$ and $\mathrm{p} 65$ expressions vere decreased after sh-GAS5 or miR-203a mimic transfection, and were increased by miR-203a inhibitor transfection. The up-regulations of phosphorylated IкB $\alpha$ and p65 were inhibited in miR-203a inhibitor + si-TIMP2 group compared with miR-203a inhibitor group.

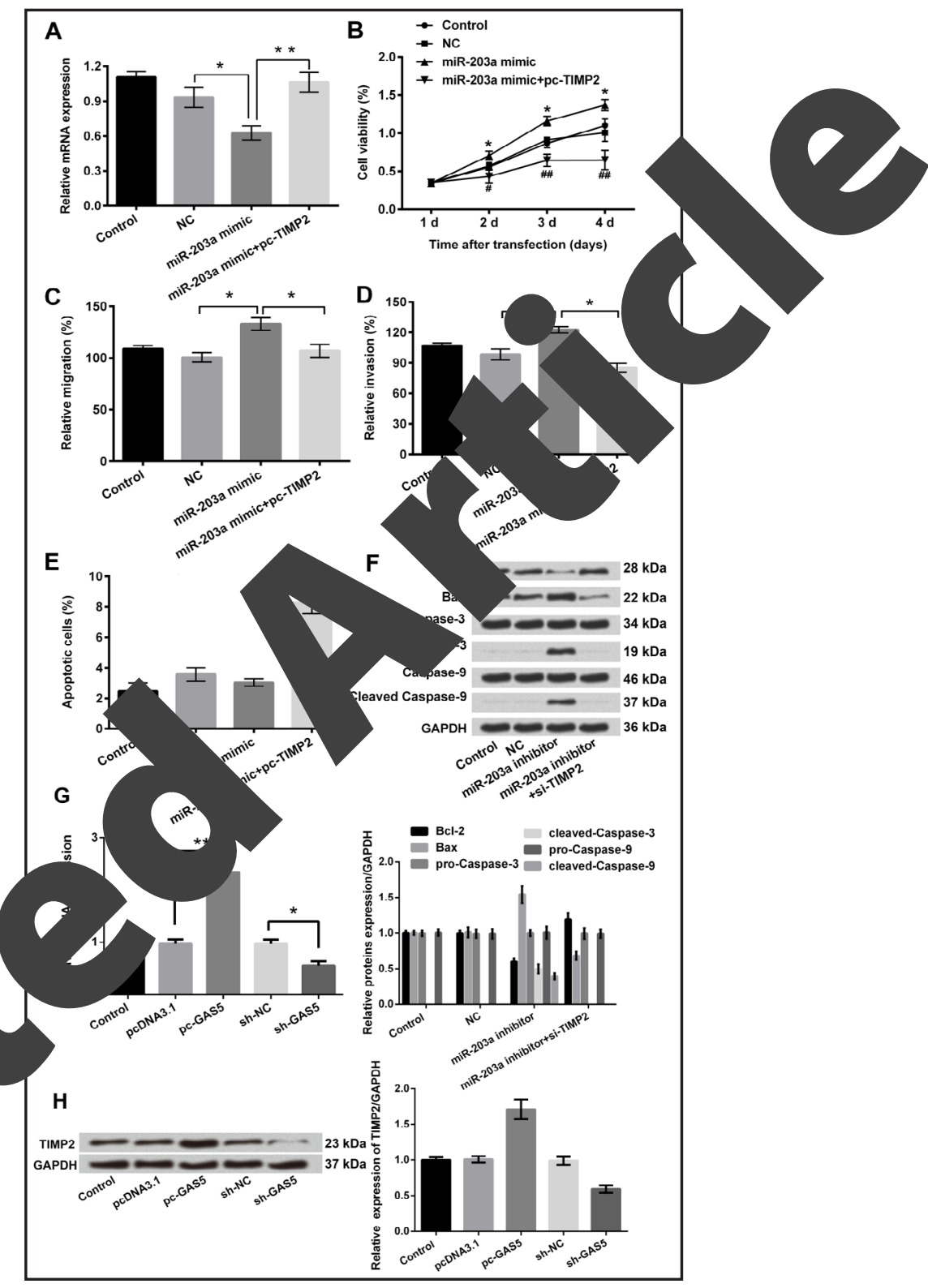

Fig. 7. Involvement of TIMP2 in miR-203a-modulated cell growth and metastasis of MG-63 cells. (A) The mRNA expression level of TIMP2 in MG-63 cells was detected by qPCR after transfection with miR-203a mimic or miR-203a mimic plus pc-TIMP2. (B) Cell viability was detected by MTT. *, P $<0.05$ comparison between miR-203a mimic and NC group. \#, P $<0.05$ and \#\#, $\mathrm{P}<0.01$ comparison between miR-203a mimic and miR-203a mimic + pc-TIMP2 group. (C) Transwell assay was performed to measure cell migration. (D) Transwell assay with matrigel was used to measure invasion. (E) Apoptotic cells were measured by flow cytometry. (F) The protein expression levels of apoptosis-related factors were measured by western blot. (G) The mRNA and (H) protein levels of TIMP2 were respectively measured by qPCR and western blot after transfection with pc-GAS5, sh-GAS5 or negative controls. *, P $<0.05$ and ${ }^{* *}, \mathrm{P}<0.01$ compared to the indicated group. 

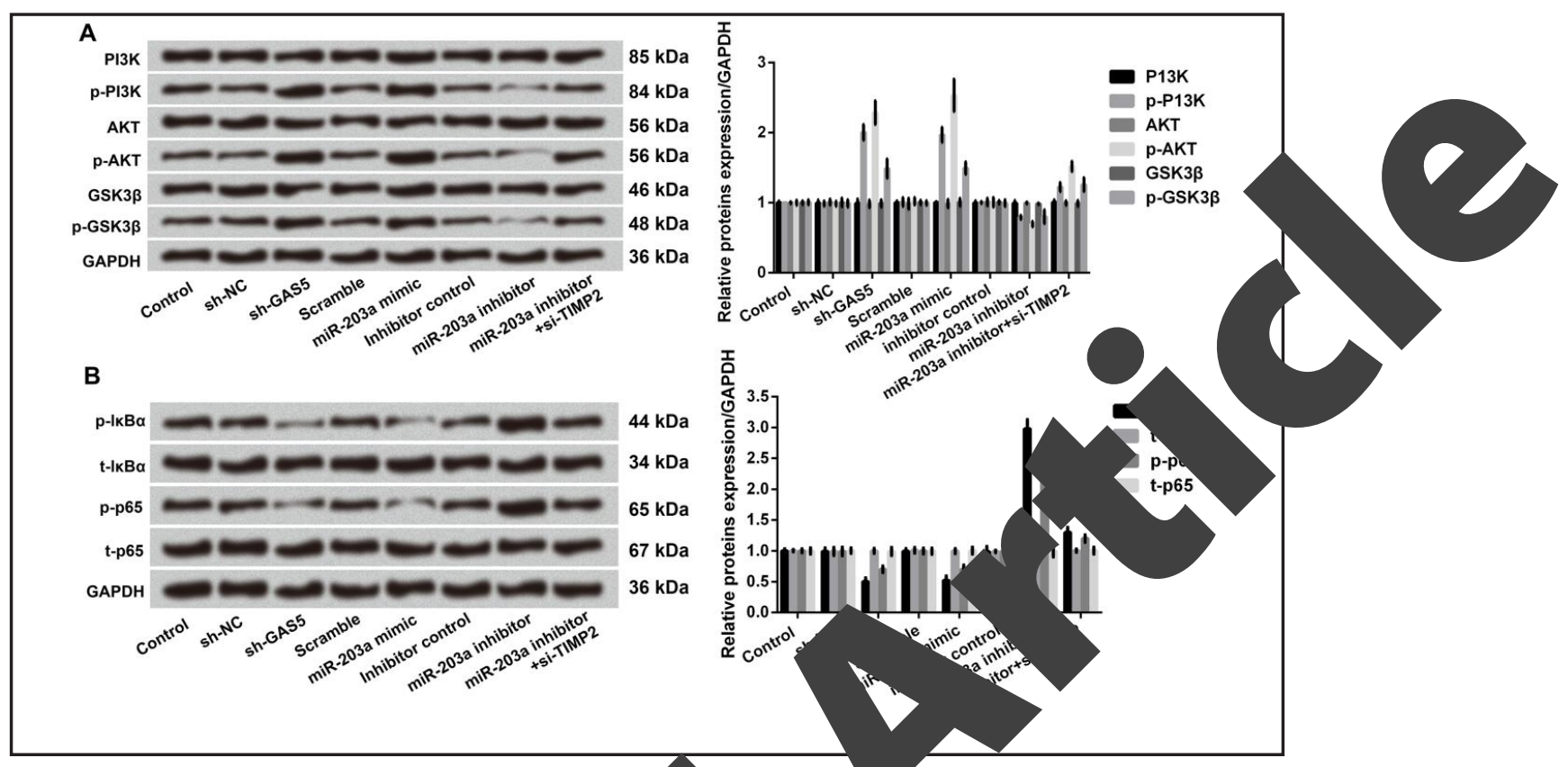

Fig. 8. Regulatory roles of IncRNA GAS5, miR-203a pathways. (A) Western blot analytical results demonst the phosph plated levels of PI3K, AKT and GSK3 $\beta$ in MG-63 cells after transfection with sh-GAS5 mimic, miR-203a inhibitor, miR-203a inhibitor plus si-TIMP2, or negative controls. (B) Weste
ylated levels of $\mathrm{I} \kappa \mathrm{B} \alpha$ and $\mathrm{p}-65$ after transfection.

\section{Discussion}

In recent decades, the ent onsarcoma has been greatly improved benefit from novel adjuvant theraner adjuvant chemotherapy [15]. However, tumor metastasis is still plag of pat with osteosarcoma [1]. In-depth study on the pathogenesis and mole r meghanisms of osteosarcoma will help to improve the current dilemma of osteosarcon ht. LncRNAs have gained widespread interest in recent years [16]. The ou be involved in many human diseases, particularly in the development sion of human malignancies [17]. In the present study, we found that lncRN GAS as wn-regulated in osteosarcoma cell lines. Silence of lncRNA GAS5 in osteg ma cells significantly improved cell viability, migration and invasion in vitro the contrary, IncRNA GAS5 overexpression inhibited cell viability, migration, n a creased cell apoptosis. These results suggested a tumor suppressive role of GAS osteosarcoma cells.

l-known that IncRNA GAS5 is a growth suppressor, that it is up-regulated in T cell growth is inhibited by starvation or rapamycin treatment $[18,19]$. LncRNA

S 5 also exerts tumor suppressive functions by controlling of cell proliferation, cell-cycle, poptosis in multiple cancers including non-cell lung cancer [20], breast cancer [6] a stomach cancer [21]. Moreover, overexpression of lncRNA GAS5 in human colorectal cancer significantly repressed the proliferation both in vitro and in vivo [22]. Consistence with the current understanding of lncRNA GAS5 in cancer cells, our study found that silence of lncRNA GAS5 promoted MG-63 cells viability, migration and invasion. The findings could enrich the knowledge regarding the roles of IncRNA GAS5 in osteosarcoma cells and provide new insights on lncRNA GAS5 as a novel tumor suppressor in tumorigenesis.

Accounting evidence displays that IncRNAs could regulate gene expression at transcriptional, post-transcriptional and epigenetic levels $[16,23]$. In order to investigate the mechanism of IncRNA GAS5 in osteosarcoma cells growth and metastasis, the interaction 


\section{Cellular Physiology Cell Physiol Biochem 2018;45:844-855

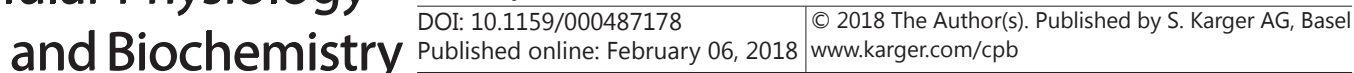 \\ Wang et al.: Effect of LncRNA GAS5 on Osteosarcoma Cells}

between IncRNA GAS5 and its target miR-203a was detected in this study. We found that miR-203a was negatively regulated by lncRNA GAS5, that silence of IncRNA GAS5 increased expression level of miR-203a. Inhibition of miR-203a in MG-63 cells reversed the effect of IncRNA GAS5 silence on cells growth and metastasis, suggesting the anti-tumor actions of IncRNA GAS5 in MG-63 cells possibly by repressing the expression of miR-203a.

Studies have proved that miRNAs act as oncogenes or suppressors to regulate expressions of human cancer genes [12]. We further studied the target gene of miR-203a i MG-63 cells. We found that miR-203a directly targeted TIMP2 and regulated the express of TIMP2. It has been reported that TIMP2 inhibited the most lethal cancer cell-metas thereby alleviating the pain caused by surgery, radiotherapy and chemot treatment of osteosarcoma [24]. Furthermore, TIMP2 has been proved to be brain metastasis of breast cancer [25] and invasive behavior of pancreat this study, we found that TIMP2 was a target of miR-203a, and overexp, had inhibitory effects on MG-63 cells growth and metastasis. Above data TIMP2 might act as a tumor suppressor, and the interaction between $n$ was related with osteosarcoma cells growth and metastasis.

To further understand the role of IncRNA GAS5 in dulating sarcoma cells growth and metastasis, the potential related molecular had investigated. We observed that IncRNA GAS5 silence and miR-203a ove re reated PI3K/AKT/

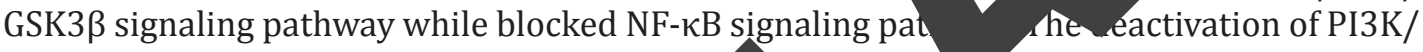
AKT/GSK3 $\beta$ signaling pathway and the activa of NF- $\kappa B$ gling pathway induced by miR-203a suppression were reserved by TIM lence. GS is a downstream target molecule of PI3K/AKT pathway that induces is through regulation of apoptosisrelated proteins [27]. GSK-3 $\beta$ was phosphor ed the signaling pathway of PI3K/ AKT is activated. NF- $\mathrm{KB}$ is found to be invol in $\mathrm{c}$ ar processes and to control DNA transcription in almost all animal cell tregulated NF- $\mathrm{\kappa B}$ is linked to cancer, inflammation, autoimmune disease, a $[29,50]$. Herein, our results indicated that lncRNA GAS5 acted as a tumar supp
pathway" involving PI3K/AK

In this study, in vitro e suppressor in osteosarcor yra mechanism underlies th 203a, sequestering miF 作 signaling and activating gnaling. There still lacks in vivo data to cross-check the function of lncP in osteosarcoma. More effort is required for clinical application of IncRNA GAS5 of $\mathrm{O}$ ma treatment. 


\section{Cellular Physiology Cell Physiol Biochem 2018;45:844-855 \begin{tabular}{ll|l|l|l|l} 
DOI: 10.1159/000487178 & 2018 The Author(s). Published by S. Karger AG, Basel \\
and Biochemistry & Published online: February 06, 2018 & www.karger.com/cpb
\end{tabular} \\ Wang et al.: Effect of LncRNA GAS5 on Osteosarcoma Cells}

6 Pickard MR, Williams GT: Regulation of apoptosis by long non-coding RNA GAS5 in breast cancer cells: implications for chemotherapy. Breast Cancer Res Treat 2014;145:359-370.

7 Cao S, Liu W, Li F, Zhao W, Qin C: Decreased expression of IncRNA GAS5 predicts a poor prognosis in cervical cancer. Int J Clin Exp Pathol 2014;7:6776-6783.

8 Lu X, Fang Y, Wang Z, Xie J, Zhan Q Deng X, Chen H, Jin J, Peng C, Li H, Shen B: Downregulation of gas5 increases pancreatic cancer cell proliferation by regulating CDK6. Cell Tissue Res 2013;354:891-896. Cao MX, Jiang YP, Tang YL, Liang XH: The crosstalk between IncRNA and microRNA in cancer metastasis: orchestrating the epithelial-mesenchymal plasticity. Oncotarget 2017;8:12472-12483.

10 Nd DG, Matkovich SJ: Menage a Trois: intimate relationship among a microRNA, long noncoding RNA, mRNA. Circ Res 2014;114:1362-1365.

11 Cesana M, Cacchiarelli D, Legnini I, Santini T, Sthandier O, Chinappi M, Tramontano A, Bozzoni noncoding RNA controls muscle differentiation by functioning as a competing endoge 2011;147:358-369.

12 Shenouda SK, Alahari SK: MicroRNA function in cancer: Oncogene or a tumor suppr Metastasis Rev 2009;28:369-378.

13 Livak KJ, Schmittgen TD: Analysis of Relative Gene Expression Data Using Rear the $2-\Delta \Delta$ C T Method. Methods 2001;25:402-408.

14 Shin SS, Keshavjee S, Gelmanova IY, Atwood S, Franke MF, Mishu AD, Barnashov A, Tonkel TP, Cohen T.: Development of extensively multidrug-resistant tuberculosis treatment. Am J Respir Crit Care $\mathrm{M}$ Bacci G, Longhi A, Versari M, Mercuri M, Briccoli A, extremity treated with neoadjuvant chemotherapy: institution. Cancer 2006;106:1154-1161.

16 Rinn JL, Chang HY: Genome regulation by long non

17 Esteller M: Non-coding RNAs in human disease. N

Mourtadamaarabouni M, Hasan AM, Farza by mammalian target of rapamycin (mT transcript 5 (GAS5). Mol Pharmacol 201 carcinoma. Asian Pac J Cancer

-20 Shi X, Sun M, Liu H, Yao Y, proliferation and apopto

-21 Liu Y, Jing Z, Zhang W, C to YBX1 to regulate $\mathrm{p} 21$

-22 Yin D, He X, Z predicts a $\$ 23$ Vadaie Morr

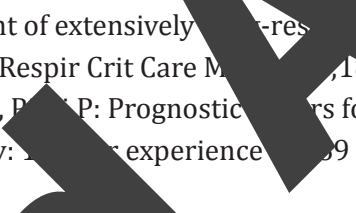
$102,26-432$.
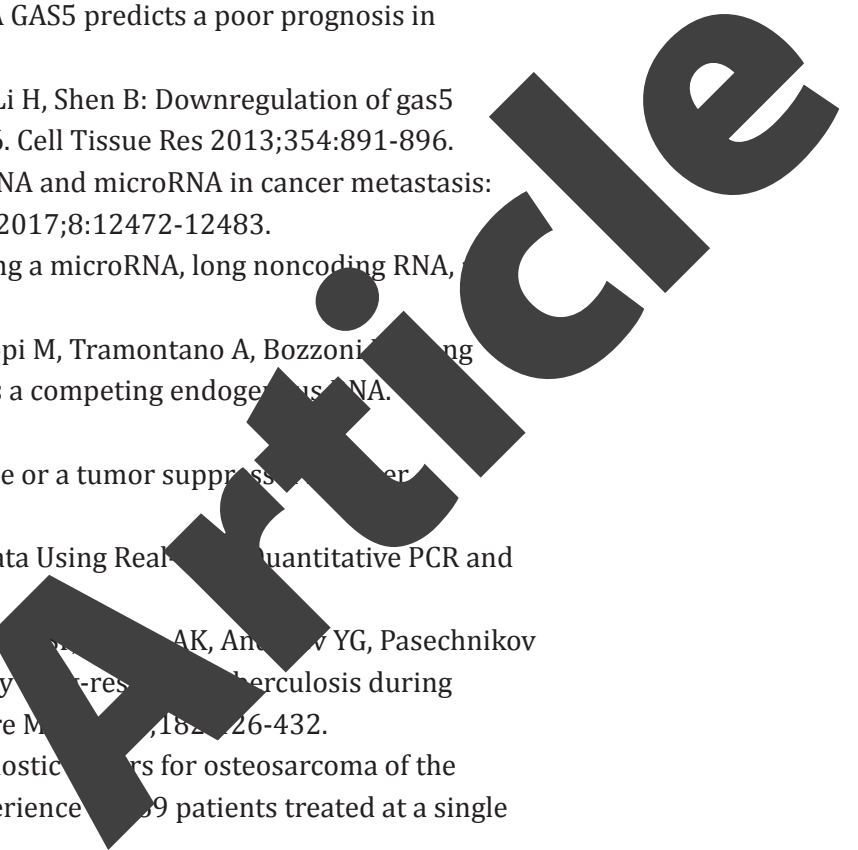
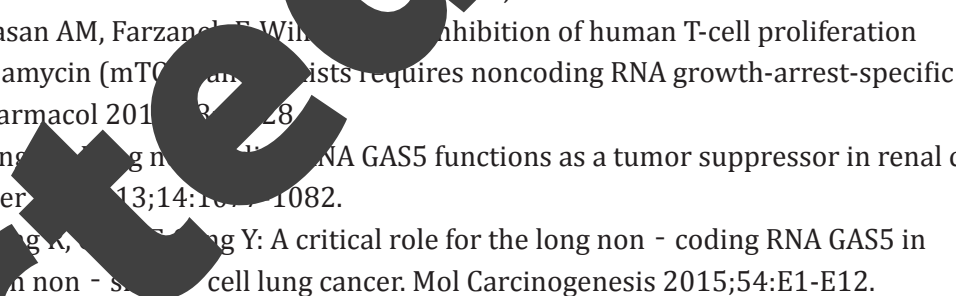
non 1082. Gent nu Rev Biochem 2012;81:145-166. 1;12:861-874.

hibition of human T-cell proliferation g Y: A critical role for the long non - coding RNA GAS5 in cell lung cancer. Mol Carcinogenesis 2015;54:E1-E12.

\section{Hu C, Yvang G, Ying Z: IncRNA GAS5 enhances G1 cell cycle arrest via binding} A stomach cancer. Sci Rep 2015;5:10159.

ng $k,-\checkmark W$, Zhang Z: Long noncoding RNA GAS5 affects cell proliferation and patients with colorectal cancer. Med Oncol 2014;31:1-8.

antisense non-coding RNAs and the epigenetic regulation of gene expression. 4:411-415.

Zhang E, Kong R, De W, Zhang Z: Long noncoding RNA GAS5 affects cell proliferation and edict. $\quad$ prognosis in patients with colorectal cancer. Med Oncol 2014;31:1-8.

ndes 0, M HT, Lungu G, Stoica G: MMP2 role in breast cancer brain metastasis development and its $\lambda$ by TIMP2 and ERK1/2. Clin Exp Metastasis 2007;24:341-351.

Rigy n, Lemoine NR: Adenoviral delivery of TIMP1 or TIMP2 can modify the invasive behavior of ancreatic cancer and can have a significant antitumor effect in vivo. Cancer Gene Ther 2001;8:869-878. Aermida MA, Dinesh Kumar J, Leslie NR: GSK3 and its interactions with the PI3K/AKT/mTOR signalling network. Adv Biol Regul 2017;65:5-15.

Panday A, Inda ME, Bagam P, Sahoo MK, Osorio D, Batra S: Transcription Factor NF-kappaB: An Update on Intervention Strategies. Arch Immunol Ther Exp (Warsz) 2016;64:463-483.

29 Zhou R, Hu G, Gong AY, Chen XM: Binding of NF-kappaB p65 subunit to the promoter elements is involved in LPS-induced transactivation of miRNA genes in human biliary epithelial cells. Nucleic Acids Res 2010;38:3222-3232.

-30 Haddad JJ, Abdelkarim NE: NF- $\kappa$ B cellular and molecular regulatory mechanisms and pathways: therapeutic pattern or pseudoregulation? Cell Immunol 2011;271:5-14. 